\title{
Airway epithelial interferon response to SARS-CoV-2 is inferior to rhinovirus and heterologous rhinovirus infection suppresses SARS-CoV-2 replication
}

Elizabeth R. Vanderwall ${ }^{1}$, Kaitlyn A. Barrow ${ }^{1}$, Lucille M. Rich ${ }^{1}$, David F. Read ${ }^{2}$, Cole Trapnell ${ }^{2}$, Oghenemega Okoloko ${ }^{1}$, Steven F. Ziegler ${ }^{3}$, Teal S. Hallstrand ${ }^{4}$, Maria P. White ${ }^{1}$, Jason S. Debley $^{1,5}$

${ }^{1}$ Center for Immunity and Immunotherapies. Seattle Children's Research Institute, Seattle, WA., USA.

${ }^{2}$ Department of Genome Sciences. University of Washington, Seattle, WA., USA.

${ }^{3}$ Center for Fundamental Immunology, Benaroya Research Institute at Virginia Mason, Seattle, Washington; Department of Immunology, University of Washington School of Medicine, Seattle, Washington.

${ }^{4}$ Division of Pulmonary, Critical Care, and Sleep Medicine and the Center for Lung Biology, University of Washington, Seattle, WA., USA.

${ }^{5}$ Department of Pediatrics, Division of Pulmonary and Sleep Medicine, Seattle Children's Hospital, University of Washington, Seattle, WA., USA.

Corresponding Author: Jason.debley@seattlechildrens.org; Jason S. Debley, MD, MPH, ORCID ID: https://orcid.org/0000-0001-6438-9535, ADDRESS: Center for Immunity and Immunotherapies, Seattle Children's Research Institute, 1900 Ninth Ave., Seattle, WA, 98145

Author Contributions: Conceptualization, K.A.B., C.T., J.S.D.; methodology, K.A.B, E.R.V., L.M.R., O.O., J.S.D.; validation, E.R.V., L.M.R., K.A.B., J.S.D.; formal analysis, E.R.V., J.S.D.; investigation, E.R.V, L.M.R, K.A.B., M.P.W., O.O., J.S.D.; resources, T.S.H., J.S.D.; data curation, O.O., K.A.B., L.M.R., M.P.W., E.R.V., J.S.D.; writingoriginal draft preparation, E.R.V, J.S.D.; writing-review and editing, E.R.V., L.M.R., M.P.W., O.O., S.F.Z., T.S.H., D.F.R., C.T., K.A.B., J.S.D.; supervision, J.S.D.; project administration, J.S.D.; funding acquisition, S.F.Z, J.S.D. All authors have read and agreed to the published version of the manuscript.

Supported by: NIH NIAID K24AI150991-01S1 (JSD); U19AI125378-05S1 (SFZ, JSD)

Word Count: Body: 4287; Abstract: 254

Key words: SARS-CoV-2, COVID-19, rhinovirus, airway epithelial cells, epithelium, interferon, children, adults 


\section{ABSTRACT}

Introduction: Common alphacoronaviruses and human rhinoviruses (HRV) induce type I and III interferon (IFN) responses important to limiting viral replication in the airway epithelium. In contrast, highly pathogenic betacoronaviruses including SARS-CoV-2 may evade or antagonize RNA-induced IFN I/III responses. Methods: In airway epithelial cells (AECs) from children and older adults we compared IFN I/III responses to SARS-CoV-2 and HRV-16, and assessed whether pre-infection with HRV-16, or pretreatment with recombinant IFN- $\beta$ or IFN- $\lambda$, modified SARS-CoV-2 replication. Bronchial AECs from children (ages 6-18 yrs.) and older adults (ages 60-75 yrs.) were differentiated ex vivo to generate organotypic cultures. In a biosafety level 3 (BSL-3) facility, cultures were infected with SARS-CoV-2 or HRV-16, and RNA and protein was harvested from cell lysates 96 hrs. following infection and supernatant was collected 48 and 96 hrs. following infection. In additional experiments cultures were pre-infected with HRV-16, or pre-treated with recombinant IFN- $\beta 1$ or IFN- $\lambda 2$ before SARS-CoV-2 infection. Results: Despite significant between-donor heterogeneity SARS-CoV-2 replicated 100 times more efficiently than HRV-16. IFNB1, INFL2, and CXCL10 gene expression and protein production following HRV-16 infection was significantly greater than following SARS-CoV-2. IFN gene expression and protein production were inversely correlated with SARS-CoV-2 replication. Treatment of cultures with recombinant IFN $\beta 1$ or IFN $\lambda 2$, or pre-infection of cultures with HRV-16, markedly reduced SARSCoV-2 replication. Discussion: In addition to marked between-donor heterogeneity in IFN responses and viral replication, SARS-CoV-2 elicits a less robust IFN response in primary AEC cultures than does rhinovirus, and heterologous rhinovirus infection, or treatment with recombinant IFN- $\beta 1$ or IFN- $\lambda 2$, markedly reduces SARS-CoV-2 replication. 


\section{INTRODUCTION}

The novel coronavirus SARS-CoV-2 has rapidly infected humans across the globe, causing one of the most devastating pandemics in modern history, with over 240 million confirmed cases and nearly 5 million deaths worldwide by October 2021(1). While most cases of the resulting coronavirus disease 2019 (COVID-19) are mild, some cases are severe and complicated by respiratory and multi-organ failure(2), with a fatality rate ranging from as low as $0.2 \%$ to as high as $27 \%$ depending on underlying medical co-morbidity and age(3). For the first half of the pandemic, incidence of COVID-19 was surprisingly low among children(3), however,

9 there is evidence that SARS-CoV-2 infection rates are as high in children as older adults(3) and that children can shed SARS-CoV-2 while asymptomatic and for prolonged periods(4). More recently, the incidence of COVID-19 in the United States has increased significantly among children and adolescents(5). Understanding mechanisms that explain the heterogeneity of severity with SARS-CoV-2 infection between individuals and across different age groups may assist efforts to develop therapeutic interventions to treat and prevent COVID-19. differences in the innate immunity between individuals, particularly the heterogeneity of type I

17 and III interferon (IFN) responses. Innate immune sensing of coronaviruses, including SARSCoV-2, is thought to occur primarily through pattern recognition receptors (PRRs) including the cytosolic RIG-I-like receptors, melanoma differentiation-associated protein 5 (MDA5; coded for by the gene IFIH1), and retinoic acid-inducible gene I (RIG-I) as well as cell surface or endosomal transmembrane toll-like receptors (TLRs) TLR3 and TLR7, which lead to the

22 activation of signaling cascades that further induce type I and III IFN responses(6) (7) (8) (9).

23 Common human coronavirus (HCoV) strains (e.g. alpha-coronavirus strain 229E) potently

24 induce type I and III IFN, and their replication is susceptible to inhibition by IFN I/III, leading to 25 suppression of the early phase of viral replication(10) (11). In contrast, previous highly 
pathogenic beta-HCoVs (e.g. SARS-CoV and MERS-CoV) encode viral proteins with a greater capability to antagonize RNA-induced type I and III IFN production through perturbation of RNA sensing(12) (13) (14) (15) (16) (17). Likewise, IFN responses at mucosal surfaces appear to be muted during SARS-CoV-2 infection as compared to other respiratory viruses, suggesting evasion of innate immune responses by SARS-CoV-2(18) (19). Data from our lab and others indicates that epithelial infection with human rhinovirus increases the expression of the entry receptors for SARS-CoV-2(20) (21), suggesting that when these two viruses concurrently infect individuals the response to one virus could modulate the response to the other.

Data from clinical studies increasingly support a hypothesis that deficiency of initial IFN responses to SARS-CoV-2 may allow for increased viral replication that then supports systemic inflammatory responses that contribute to COVID-19 pathology and severity(19) (22) (23) (24). Ziegler et al. recently performed scRNA-seq on nasopharyngeal swabs from 15 healthy adults, 14 adults with mild COVID-19 and 21 adults with severe COVID-19, and observed that epithelial cells from patients with severe COVID-19 had less robust expression of anti-viral interferon response genes as compared to patients with mild COVID-19 and healthy controls supporting their conclusion that a "failed" nasal epithelial innate anti-viral response may be a risk factor for severe COVID-19(25).

The objectives of our study were to determine if heterogeneity in bronchial epithelial type I and III IFN responses to SARS-CoV-2 between individual pediatric and adult donors was associated with SARS-CoV-2 replication, to compare airway epithelial IFN responses between SARS-CoV-2 and human rhinovirus-A16 (HRV-16), and to determine the effects of HRV preinfection or exogenous IFN treatment on SARS-CoV-2 replication in organotypic airway epithelial cell (AEC) cultures from children and adults. We hypothesized that type I and III IFN responses would be less vigorous to SARS-CoV-2 than to HRV infection, that IFN responses 
51 recombinant IFN treatment of airway epithelial cultures would decrease replication of SARS-

52 CoV-2. Some of the results of these studies have been previously reported in the form of an

53 abstract(26).

54

55 METHODS

Bronchial AECs from children ages 6-18 years $(n=15)$ and older adults ages 60-75 years

$57(n=10)$ were differentiated ex vivo at an air-liquid interface (ALI) to generate organotypic

58 cultures. AECs from children were obtained under study \#12490 approved by the Seattle

59 Children's Hospital Institutional Review Board. Parents of subjects provided written consent and

60 children over 7 years of age provided assent. Primary bronchial AECs from adults were

61 purchased from Lonza® or obtained from a tracheal segment lung transplant donor lung tissue.

62 AECs were differentiated ex vivo for 21 days at an ALI on 12-well collagen-coated Corning®

63 plates with permeable transwells in PneumaCult ${ }^{\mathrm{TM}} \mathrm{ALI}$ media (Stemcell ${ }^{\mathrm{TM}}$ ) at $37^{\circ} \mathrm{C}$ in an

64 atmosphere of $5 \%$ CO2 as we have previously described, producing an organotypic

65 differentiated epithelial culture with mucociliary morphology(27) (28) (29) (30).

67 alone, infection of AECs with HRV-16 alone, infection of AECs with HRV-16 followed by

68 infection with SARS-CoV-2 72 hours later, infection of IFN 1 1 treated AECs with SARS-CoV-2,

69 and infection of IFN $\lambda 2$ treated AECs with SARS-CoV-2. For AECs treated with recombinant IFN,

70 recombinant IFN $\beta 1(1 \mathrm{ng} / \mathrm{mL})$ or IFN $\lambda 2(10 \mathrm{ng} / \mathrm{mL})$ was added to basolateral transwell chamber

71 with every medium change, starting 72 hours prior to SARS-CoV-2 infection and continuing until

7296 hours following SARS-CoV-2 infection. The concentrations of recombinant IFN $\beta 1$ and IFN $\lambda 2$

73 were chosen based on data from preliminary experiments in three primary AEC lines comparing

74 the effect of a range of concentrations of each cytokine from $0.1-10 \mathrm{ng} / \mathrm{mL}$ on SARS-CoV-2 
replication (data not shown). In a Biosafety Level 3 (BSL-3) facility, cultures were infected with SARS-CoV-2 isolate USA-WA1/2020 or HRV-16 at a multiplicity of infection (MOI) of 0.5. At 96 hrs. following SARS-CoV-2, or following HRV-16 infection alone, RNA was isolated from cells using Trizol® and protein was isolated from cell lysates with RIPA buffer (Sigma-Aldrich®) containing Triton X100 $1 \%$ and SDS $0.1 \%$, methods that we have demonstrated fully inactivate SARS-CoV-2(31).

Expression of IFNB1, IFNL2, CXCL10, IFIH1, and GAPDH were measured by quantitative polymerase chain reaction (qPCR) using Taqman® probes. To measure SARSCoV-2 replication in AEC cultures we used the Genesig® Coronavirus Strain 2019-nCoV Advanced PCR Kit (Primerdesign $\AA$ ), with duplicate assays of harvested RNA from each SARSCoV-2-infected AEC experimental condition. The viral copy number used in analyses of each experimental condition was the mean of duplicate assays from each experimental condition. Similarly, to measure HRV-16 replication in AEC cultures we used the Genesig® Human Rhinovirus Subtype 16 PCR Kit (Primerdesign®).

To extract protein from the cell layer of SARS-CoV-2-infected AEC cultures, media was first removed from the basolateral chamber of transwells. Next, $100 \mu \mathrm{L}$ of cold PBS was added to the apical surface of cultures and $1 \mathrm{~mL}$ was added to the basolateral chamber of cultures as a wash step. Next, $50 \mu \mathrm{L}$ of RIPA buffer for protein extraction ready-to-use-solution (SigmaAldrich $\AA$, Product No. R0278) containing Triton X100 $1 \%$ and SDS $0.1 \%$ was added to the apical surface of AECs and incubated for 15 minutes on ice. A pipet tip was then used to gently scratch each apical well in a crosshatch pattern to loosen AECs from the transwell membrane. Material was collected, centrifuged at $10,000 \mathrm{rpm}$ at $4^{\circ} \mathrm{C}$ for 10 minutes, then supernatant containing isolated protein was collected. IFN $\beta 1$, IFN $\lambda 2$, and CXCL-10 protein concentrations in cell lysates, and IFN $\beta 1$, IFN $\lambda 3$, and $C X C L-10$ concentrations were measured in cell culture 
supernatants, via a Human Luminex® Assay $(R \& D \AA)$, with protein concentrations normalized to total protein levels in lysate (BCA assay; Sigma-Aldrich®).

\section{Statistical Analysis}

Gene expression and protein levels are presented as means +/- standard deviation (SD) when data were normally distributed, and as medians with interquartile range if one or more groups were not normally distributed. To determine if data was normally distributed the Kolmogorov-Smirnov test was used (alpha $=0.05)$. IFNB1, IFNL2, IFIH1 and CXCL10 relative expression were standardized using GAPDH as a non-regulated housekeeping gene. GenEx version 5.0.1 was used to quantify gene expression from qPCR normalized to GAPDH (MultiD Analyses AB, Göteborg, Sweden) based on methods described by Pfaffl(32). Data in at least one group or condition in each experiment analyzed were determined to be non-normally distributed, therefore nonparametric tests were used for analyses. To compare gene expression data and distributions of protein concentrations in cell lysates and supernatants between paired groups the Wilcoxon matched-pairs signed rank test was used. For unpaired data the MannWhitney test was used for analyses. For experiments with three or more conditions the KruskalWallis one-way ANOVA on ranks test was used, and post hoc comparisons between pairs of subject groups were made using Dunn's multiple comparisons test (significance level set at $\mathrm{p}<0.05)$. Correlations were determined using the Spearman's rank correlation coefficient. Data was analyzed using Prism® 9.0 software (GraphPad Software Inc., San Diego, CA.). Statistical significance was set at $p<0.05$.

\section{RESULTS}

In organotypic primary bronchial AEC cultures from children $(n=15)$ and older adults $(n=10)$ we observed marked heterogeneity in SARS-CoV-2 replication between human donors 
123 (Figure 1). The clinical characteristics of human airway epithelial donors included in these

124 experiments is summarized in Table 1. Despite the significant between-subject heterogeneity in

125 SARS-CoV-2 replication, we observed that SARS-CoV-2 replicated approximately 100 times

126 more efficiently than HRV-16 in these primary bronchial AEC cultures (Figure 1; SARS-CoV-2

127 median copy number 215,387 vs. HRV-16 median copy number $2211 ; \mathrm{p}<0.0001)$ when parallel

128 cultures from each donor were infected with each virus at the same $\mathrm{MOI}$ of 0.5 . When data

129 from pediatric and adult cultures were analyzed separately SARS-CoV-2 replication was also

130 markedly greater than HRV-16 in cultures within each donor age group (children: SARS-CoV-2

131 median copy number 215,387 vs. HRV-16 median copy number 2602 ; $p<0.001$; adults: SARS-

132 CoV-2 median copy number 75,940 vs. HRV-16 median copy number $2184 ; p=0.002$ ). SARS-

133 CoV-2 replication was not significantly different between AEC cultures from pediatric and adult 134 donors (median copy number 215,387 vs. 75,$940 ; p=0.23$ ), and among pediatric donors SARSCoV-2 replication was not significantly different between cultures from children with asthma and healthy children (median copy number 60,540 vs. 436,$465 ; p=0.3$ ). compared in parallel, RNA harvested 96 hours following infection was available from 22 donor cultures ( $\mathrm{n}=14$ children, $\mathrm{n}=8$ adults) to allow measurement of IFNB1, IFNL2, and CXCL10 gene expression, and protein was available from cell lysate collected 96 hours following infection from 20 donor cultures ( $n=12$ children, $n=8$ adults) to allow for measurement of IFN $\beta 1$, IFN $\lambda 2$ (IL28A), and CXCL-10 protein levels. As compared to uninfected cultures, the relative increase in expression of IFNB1 following infection with HRV-16 was significantly greater than following

144 infection with SARS-CoV-2 (median increase expression 4.4-fold vs. 1.4-fold, $\mathrm{p}<0.0001$; Figure 1452 , panel A). Similarly, the relative increase in expression of IFNL2 following infection with HRV-

14616 was significantly greater than following infection with SARS-CoV-2 (median increase

147 expression 21.2-fold vs. 4.3-fold, p<0.0001; Figure 2, panel C), as was the increase in 
148

149

150

expression of CXCL10 (median increase expression 9.8-fold vs. 5.4-fold, $\mathrm{p}=0.003$; Figure 2, panel E). The expression of these three genes was significantly greater following HRV-16 infection than following SARS-CoV-2 in cultures from both children and adults when analyzed separately (data not shown). The concentrations of IFN $\beta 1$, IFN $\lambda 2$ (IL-28A), and CXCL-10 protein, normalized to total protein concentration, in cell lysates collected 96 hours following infection with HRV-16 were also significantly greater than in parallel cultures following SARSCoV-2 infection (IFNß1: median 892 vs. 663 pg/mL, p=0.02, Figure 2, panel B; IFN $\lambda 2$ (IL-28A): 9848 vs. 7123 pg/mL, p=0.02, Figure 2, panel D; and CXCL-10: 69,306 vs. 15,232 pg/mL, $\mathrm{p}<0.0001$, Figure 2, panel F).

Of cultures wherein SARS-CoV-2 and HRV-16 infection was compared in parallel, supernatant was collected from $n=16$ donor cell lines 48 hours following infection and from $n=20$ cell lines 96 hours following infection. Concentrations of IFN $\beta 1$ in supernatant (normalized to total protein concentration) were higher at 48 hours vs. 96 hours post infection for both viruses. However, IFN $\beta 1$ concentrations were significantly greater following HRV-16 as compared to SARS-CoV-2 infection at both 48 hours (median 60.4 vs. 12.5 pg/mL, $p<0.001$, Figure 3, panel A) and 96 hours (median 7.1 vs. 1.4 pg/mL, p<0.001, Figure 3, panel A). IFN $\lambda 2$ (IL-28A) concentrations were below the assay detection level in supernatants for most samples (data not shown). IFN $\lambda 3$ (IL-28B) concentrations in supernatants were significantly greater following HRV16 as compared to SARS-CoV-2 infection at both 48 hours (median 1335 vs. $40.6 \mathrm{pg} / \mathrm{mL}$, $\mathrm{p}<0.001$, Figure 3, panel B) and 96 hours (median 197 vs. 48 pg/mL, $p<0.001$, Figure 3, panel B). CXCL10 concentrations in supernatants were also significantly greater following HRV-16 as compared to SARS-CoV-2 infection at both 48 hours (median 293,805 vs. 10,407 pg/mL, $\mathrm{p}<0.001$, Figure 3, panel C) and 96 hours (median 179,858 vs. 150,939 pg/mL, $\mathrm{p}=0.04$, Figure 3, panel C). 
At 96 hours following infection we assessed correlations between relative expression of IFNB1 and IFNL2 in individual primary bronchial epithelial cell lines and viral replication (SARS174 CoV-2 copy number) in those cultures. Both IFNB1 and IFNL2 gene expression was inversely 175 correlated with SARS-CoV-2 replication (IFNB1 $\mathrm{r}=-0.61, \mathrm{p}=0.003$; IFNL2 $\mathrm{r}=-0.42, \mathrm{p}=0.05$; Figure 176 4). Because concentrations of IFN $\beta 1$ and IFN $\lambda 3$ in supernatants were highest at 48 hours 177 following infection, we assessed correlations between supernatant concentrations of these 178 cytokines at 48 hours following SARS-CoV-2 infection and viral replication at 96 hours following 179 infection and observed a significant inverse correlation between supernatant IFN $\beta 1$ 180 concentrations and viral replication $(r=-0.53, p=0.02$; Figure 5, panel $A)$ and a trend toward an inverse correlation between supernatant IFN $\lambda 3$ concentrations and viral replication $(r=-0.44$, 182 $\mathrm{p}=0.06$, data not shown). We observed significant negative correlations between $\mathrm{CXCL} 10$ protein concentrations in both supernatant $(r=-0.56, p=0.01$, data not shown) and cell lysate $(r=-$ 0.65, $p=0.002$; Figure 5, panel B) at 96 hours following infection and SARS-CoV-2 replication. In organotypic bronchial epithelial cultures from 14 children and 10 older adults, replication of SARS-CoV-2 was compared between cultures infected with SARS-CoV-2 alone $187(\mathrm{MOI}=0.5)$, infection of cultures with HRV-16 $(\mathrm{MOI}=0.5)$ followed 72 hours later by infection with SARS-CoV-2 (MOI=0.5), infection of IFNß1 pre- and concurrently treated cultures with SARSCoV-2, and infection of IFN $\lambda 2$ pre- and concurrently treated cultures with SARS-CoV-2. Preinfection of bronchial AECs with HRV-16 led to a marked reduction in SARS-CoV-2 replication 96 hours following infection (median SARS-CoV-2 copy number 267,264 vs. 14,788, p=0.002;

192 Figure 6). Treatment of AEC cultures with recombinant IFN $\beta 1$ reduced SARS-CoV-2 replication 193 from a median copy number of 267,264 to $11,947(p=0.0001)$ and treatment of AEC cultures 194 with recombinant IFN $\lambda 2$ reduced SARS-CoV-2 replication from a median copy number of 195267,264 to $11,856(p=0.0002)$. 

various steps between viral sensing and transcription and translational of type I and III

198 interferons, and ultimately transcription of an array antiviral genes(12) (13) (14) (15) (16) (17),

199 we assessed one potential proximal step where SARS-CoV-2 may evade sensing of viral

200 nucleic acids by comparing gene expression of the pattern-recognition receptor and RNA viral

201 sensor IFIH1/MDA5 between primary bronchial AEC cultures infected in parallel with SARS-

202 CoV-2 $(\mathrm{MOI}=0.5)$ or $\mathrm{HRV}-16(\mathrm{MOI}=0.5)$. We observed that IFIH1 expression was more than 2-

203 fold greater following infection with HRV-16 as compared to following SARS-CoV-2 infection

204 (Figure 7; $p=0.003$ ).

\section{DISCUSSION}

A growing body of literature suggests that beta-HCoVs, including SARS-CoV-2 appear able to antagonize type I and III IFN responses at mucosal surfaces at multiple steps between viral sensing and production of interferon induced antiviral proteins(18) (19) (33). In this study we directly compared type I and III IFN responses to SARS-CoV-2 and HRV-16 infection by primary organotypic bronchial AEC cultures from children and adults, and assessed the impact

212 of exogenous treatment with recombinant IFN $\beta 1$ or IFN $\lambda 2$ on SARS-CoV-2 replication as well as

213 the impact of heterologous infection with HRV-16 prior to SARS-CoV-2. We observed significant 214 heterogeneity in SARS-CoV-2 replication between primary AEC lines from different human 215 donors, however, despite between donor heterogeneity we also observed that SARS-CoV-2 216 replicated approximately 100 times more efficiently than HRV-16 in these primary bronchial

217 AEC cultures. As compared to uninfected cultures, the relative increase in expression of IFNB1, 218 INFL2, and CXCL10 following infection with HRV-16 was significantly greater than following 219 infection with SARS-CoV-2, and the protein concentrations of type I and III IFN and the IFN 
stimulated chemokine CXCL10 in both cell lysates and supernatant were significantly greater in AEC cultures following infection with HRV-16 as compared to SARS-CoV-2. In SARS-CoV-2 infected AEC cultures type I and III IFN gene expression and protein production were inversely correlated with viral replication. Furthermore, treatment of AEC cultures with recombinant IFN $\beta 1$ or IFN $\lambda 2$, or pre-infection of AEC cultures with HRV-16, markedly reduced SARS-CoV-2 replication.

Sensing of beta-HCoVs by the innate immune system is believed to be primarily through pattern recognition receptors (PRRs), including cell surface or endosomal transmembrane TLRs TLR3 and TLR7, the cytosolic RIG-I-like receptors melanoma differentiation-associated protein 5 (MDA5), as well as retinoic acid-inducible gene I (RIG-I) (6) (7) (8) (9). PRR's then mediate activation of signaling cascades leading to induction of type I and III IFN responses(6) (7) (8) (9). Recently Sampaio et al. reported that in the lung cancer cell line Calu-3 the cytosolic RNA sensor MDA5 was required for type I and III IFN induction when cells were infected with SARSCoV-2 infection(6).

Studies using immortalized cell lines (e.g. Vero, HeLa, Calu-3, 293T) in vitro, as well as murine in vivo studies, have suggested a number of potential mechanisms by which betaHCoVs (e.g. SARS-CoV, MERS-CoV, and SARS-CoV-2) may evade IFN responses at the level of the airway epithelium. Prior to the onset of the COVID-19 pandemic, these mechanisms were investigated extensively for SARS-CoV and MERS-CoV. One group of beta-HCoV proteins, the predominantly non-structural proteins (nsps), are recognized to have IFN-antagonistic impacts. Several nsps (e.g. nsp1 and nsp3) interfere with signal transduction mediated by PRRs, while other nsps evade recognition by PRRs in mucosal epithelial cells by modifying features of the viral RNA(34). There is growing evidence that SARS-CoV-2, much like SARS-CoV and MERSCoV, has evolved a number of immune evasion strategies that may interfere with PRR's themselves(35) (36) (37) (38) (39) (40) (41) (42), inhibit multiple steps in the signaling cascade 
leading to induction and translation of type I and III IFNs(43) (44) (45) (46) (47) (48) (49) (50) (51) (52), and interfere with the actions of IFNs by impeding the signaling pathways that lead to transcription and translation of anti-viral interferon stimulated genes (ISGs) (18) (53) (54) (55) (56). Lei et al. demonstrated that the SARS-CoV-2 proteins NSP1, NSP3, NSP12, NSP13, NSP14, ORF3, ORF6 and M protein all have some ability to inhibit Sendai virus-induced IFN- $\beta$ promoter activation, and that ORF6 has inhibitory effects on both type I IFN production as well as signaling downstream of IFN- $\beta$ production(18). Early in the COVID-19 pandemic Blanco-Melo et al. reported results from a transcriptome profiling study of various immortalized cell lines which demonstrated that SARS-CoV-2 infection elicited very low type I and III IFN and limited ISG responses, while inducing expression of pro-inflammatory cytokines genes(19), raising the possibility that a deficient epithelial IFN response to SARS-CoV-2 may facilitate enhanced local viral replication that ultimately might lead to a dysregulated systemic pro-inflammatory response.

Data from several clinical studies have provided additional support for the hypothesis that a muted initial local IFN response to SARS-CoV-2 in the airway epithelium, at least in some hosts, allows the virus to replicate unimpeded which then sets up the host for potential systemic inflammatory responses that contribute to COVID-19 pathology and severity(19) (22) (23) (24). Recently, Ziegler et al. published transcriptomics results from nasopharyngeal swabs from 15 healthy adults, 14 adults with mild COVID-19 and 21 adults with severe COVID-19, and observed that nasal epithelial cells from patients with severe COVID-19 exhibited less robust expression of anti-viral IFN response genes as compared to patients with mild COVID-19 and healthy adults, supporting their conclusion that a "failed" nasal epithelial innate anti-viral response may be a risk factor for severe COVID-19(25). 
clear that clinical outcomes with COVID-19 following infection with SARS-CoV-2 is heterogeneous with outcomes even in young adults and children without medical comorbidities unpredictably ranging from ranging from asymptomatic infection to death(57). An objective of our study was to determine if heterogeneity in airway epithelial IFN responses to SARS-CoV-2 between individual pediatric and adult donors was associated with SARS-CoV-2 replication. A striking observation in our data is the marked between-donor heterogeneity in the replication of SARS-CoV-2 in organotypic AEC cultures using standardized protocols and uniform viral inoculation doses. A potential important future area of investigation will be to investigate possible genetic and epigenetic factors that may partially explain heterogeneity in SARS-CoV-2 replication in airway epithelium.

Our group and others have demonstrated that the SARS-CoV-2 entry receptor ACE2 is an ISG(20) (21). We have demonstrated that HRV-16 infection induces a type I and III interferon response, and increases ACE2 expression(21), leading us to originally speculate that HRV pre-infection of AECs might increase replication of SARS-CoV-2 through greater expression of the entry receptor and be a clinical risk factor for acquisition of COVID-19. However, our results in this study demonstrate that even through the SARS-CoV-2 entry factor ACE2 is an ISG, HRV-16 infection induces a much more potent type I and III IFN responses than SARS-CoV-2 and that heterologous infection of organotypic AEC cultures with HRV-16 three days prior to inoculation with SARS-CoV-2 markedly reduces replication of SARS-CoV-2. This suppression of SARS-CoV-2 replication was similar to the effects of exogenous treatment with IFN $\beta 1$ or IFN $\lambda 2$, suggesting that the pronounced induction of these genes by HRV-16 was responsible for these findings. These findings extend upon several other recent reports including Cheemarla et al. who reported experiments in differentiated primary airway epithelial cultures from small number of adult donors and observed that infection with HRV-01A prior to infection with SARS-CoV-2 accelerated induction of ISGs and reduced SARS-CoV-2 
295

296

297

298

299

300

301

302

303

304

305

306

307

308

replication(58). Similarly, Dee et al. used differentiated primary airway epithelial cultures from a single human donor, to characterize viral replication kinetics of SARS-CoV-2 with and without co-infection with rhinovirus and observed that pre-infection with HRV-16A reduced SARS-CoV-2 replication(59). Neither of these prior studies included primary ALI cultures from a robust sample size of adults and children or compared interferon and ISG responses between parallel HRV and SARS-CoV-2 infections in addition to HRV pre-infection to determine if this phenomenon is consistent across donors with heterogenous interferon responses to both viruses. Although difficult to definitely test, given that the incidence of COVID-19 in children was very low in the early months of the pandemic, during the peak of the late winter viral respiratory season in the United States and Europe when rhinovirus, RSV, and influenza activity was high, our data together with the studies by Dee and Cheemarla $(58,59)$ lead us to hypothesize that high rates of typical respiratory viral pathogens among Children in the northern hemisphere in February-March 2020 may have contributed to protection of children early in the COVID-19 pandemic by generally inducing airway type I and III IFN responses.

Given the steady evolution of new SARS-CoV-2 variants through 2021 and continued significant resistance to vaccination among a sizable minority of people with access to vaccines, the pandemic has continued to result in high levels of morbidity and mortality in many areas of the world, fueling an ongoing need for therapeutics to treat COVID-19. Our results demonstrating marked reduction in SARS-CoV-2 replication in AEC cultures treated with recombinant IFN $\beta 1$ or IFN $\lambda 2$ provides further mechanistic evidence to support the possible use of inhaled interferon as a possible treatment option if initiated early enough during COVID-19. A recent randomized, double-blind, placebo-controlled, phase 2 trial of inhaled nebulized interferon beta-1a (SNG001) for treatment of SARS-CoV-2 infection demonstrated that patients who received SNG001 early in their disease course had greater odds of improvement and 
recovered more rapidly from SARS-CoV-2 infection than patients who received placebo, providing a strong rationale for further trials of this agent(60).

We are not aware of other studies to date that have directly compared innate immune responses between SARS-CoV-2 and HRV in organotypic AEC cultures from many pediatric

323 and adult donors. However, there are several limitations of our primary airway epithelial model

324 system. First, our ex vivo system lacks interaction with immune cells and the complex immune

325 responses that occur in vivo in the context of COVID-19, and therefore we cannot assess how

326 heterogeneity in interferon responses to SARS-CoV-2 at the level of the airway epithelium relate

327 to systemic immune responses or clinical outcomes in vivo. Second, in this study we did not

328 investigate potential genetic or epigenetic factors that may explain the between subject

329 heterogeneity in interferon responses and viral replication that we observed. Finally, given the

330 limitations posed by the complex logistics of completing these experiments in a biosafety level 3

331 (BSL-3) facility, together with limitations in available material from organotypic cultures from a

332 sizeable number of human donors, we were constrained in the number of feasible sample

333 harvesting timepoints which prevented us from conducting a high resolution assessment of the

334 time kinetics of viral infection and interferon responses in the present study; however, our

335 choice to harvest supernatant 48 hours following SARS-CoV-2 infection and RNA 96 hours

336 following infection was informed by both our prior work with RSV(29) and preliminary

337 experiments with SARS-CoV-2 (data not shown) where we observed that in organotypic primary

338 ALI cultures type I and III interferon responses peak between 24-48 hours while expression of

339 downstream ISGs peak between 72-96 hours.

In conclusion, in this study we have demonstrated that in addition to remarkable

341 between subject heterogeneity in interferon responses and viral replication, SARS-CoV-2 elicits

342 a less robust type I and III interferon response in organotypic primary bronchial AEC cultures 
343 than does human rhinovirus, and that pre-infection of AECs with HRV-16, or pre-treatment with

344 recombinant IFN- $\beta 1$ or IFN- $\lambda 2$, markedly reduces SARS-CoV-2 replication.

345

346

347 List of Abbreviations

348 COVID-19: coronavirus disease 2019

349 IFN: interferon

350 PRR: pattern recognition receptors

351 MDA5: melanoma differentiation-associated protein 5

352 RIG-I: retinoic acid-inducible gene I

353 TLR: toll-like receptor

354 HCoV: human coronavirus

355 HRV-16: human rhinovirus-A16

356 AEC: airway epithelial cell

357 ALI: air-liquid interface

358 BSL-3: Biosafety Level 3

359 MOI: multiplicity of infection 
qPCR: quantitative polymerase chain reaction

\section{Declarations}

Ethics Approval: Airway epithelial cells from children were obtained under study \#12490 approved by the Seattle Children's Hospital IRB. Parents of subjects provided written consent and children over 7 years of age provided assent. Airway epithelial cells from adults were purchased from Lonza® without personal identifiers. The Seattle Children's Hospital IRB determined that use of de-identified adult airway epithelial cells purchased from Lonza® did not require ethics approval or consent.

Consent for publication: This manuscript does not contain any individual person's data in any 370 form.

Availability of data and materials: The datasets used and/or analysed during the current study are available from the corresponding author on reasonable request.

Competing interests: The authors declare that they have no competing interests.

Funding: NIH NIAID K24Al150991-01S1 (JSD); U19AI125378-05S1 (SFZ, JSD)

Author Contributions: Conceptualization, K.A.B., C.T., J.S.D.; methodology, K.A.B, 376 E.R.V., L.M.R., O.O., J.S.D.; validation, E.R.V., L.M.R., K.A.B., J.S.D.; formal analysis, 377 E.R.V., J.S.D.; investigation, E.R.V, L.M.R, K.A.B., M.P.W., O.O., J.S.D.; resources, 378 T.S.H., J.S.D.; data curation, O.O., K.A.B., L.M.R., M.P.W., E.R.V., J.S.D.; writing379 original draft preparation, E.R.V, J.S.D.; writing-review and editing, E.R.V., L.M.R., 380 M.P.W., O.O., S.F.Z., T.S.H., D.F.R., C.T., K.A.B., J.S.D.; supervision, J.S.D.; project 381 administration, J.S.D.; funding acquisition, S.F.Z, J.S.D. All authors have read and 382 agreed to the published version of the manuscript. 
383 Table 1: Airway Epithelial Cell Donor Characteristics

\begin{tabular}{|l|c|c|}
\hline & $\begin{array}{c}\text { Pediatric AEC Donors } \\
(\mathbf{n = 1 5})\end{array}$ & $\begin{array}{c}\text { Adult AEC Donors } \\
(\mathbf{n = 1 0})\end{array}$ \\
\hline Age (yrs., mean +/- SD) & $10.5+/-2.0$ & $67+/-4.9$ \\
\hline Gender (female) & $9(60 \%)$ & $4(40 \%)$ \\
\hline Active Smoker & $0(0 \%)$ & $3(30 \%)$ \\
\hline History of Asthma & $8(53 \%)$ & $0(0 \%)$ \\
\hline Obesity & $0(0 \%)$ & $3(3 \%)$ \\
\hline Hypertension & $0(0 \%)$ & $4(40 \%)$ \\
\hline
\end{tabular}

384

$385 \quad$ AEC = Airway epithelial cell 
386 Figure 1. SARS-CoV-2 and HRV-16 replication by quantitative PCR in primary bronchial AECs

387 from children $(n=15)$ and adults $(n=10)$. Viral copy number was quantified by PCR in RNA

388 harvested from AEC cultures 96 hours following infection (MOI of 0.5 ) with either SARS-CoV-2

389 (red circles) or HRV-16 (blue triangles). SARS-CoV-2 replication was significantly greater than

390 HRV-16 (median copy number 215,387 vs. HRV-16 median copy number $2211 ; \mathrm{p}<0.0001$ by

391 Wilcoxon matched-pairs signed rank test; bars indicate median values). 
Figure 2. Relative gene expression of IFNB1, IFNL2, and CXCL10 (normalized to GAPDH expression) by primary bronchial airway epithelial cell cultures in children $(n=14)$ and adults

$394(\mathrm{n}=8)$, and parallel IFN $\beta 1$, IFN- $\lambda 2$ (IL-28a), and CXCL10 protein concentrations in cell lysates

395 (normalized to total protein concentration), from primary bronchial airway epithelial cell cultures 396 from children $(n=12)$ and adults $(n=8)$ harvested 96 hours after SARS-CoV-2 (red circles) or 397 HRV-16 (blue triangles) infection. Expression of IFNB1 and corresponding concentrations of 398 IFN 31 in cell lysates were significantly greater in cultures after infection with HRV-16 than in 399 cultures infected with SARS-CoV-2 (Panel A, median increase expression 4.4-fold vs 1.4-fold, $400 \mathrm{p}<0.0001$; Panel B, median $892 \mathrm{pg} / \mathrm{mL}$ vs $663 \mathrm{pg} / \mathrm{mL}, \mathrm{p}=0.02)$. Expression of IFNL2, and IFN$401 \lambda 2$ protein concentrations in cell lysates, were significantly greater following HRV-16 infection 402 than SARS-CoV-2 infection (Panel C, median increase expression 21.2-fold vs 4.3-fold, $403 \mathrm{p}<0.0001$; Panel D, median $9848 \mathrm{pg} / \mathrm{mL}$ vs $7123 \mathrm{pg} / \mathrm{mL}, \mathrm{p}=0.02$ ). Expression of $C X C L 10$, and 404 CXCL10 protein concentrations in cell lysates, were significantly greater following HRV-16 405 infection as compared to SARS-CoV-2 infection (Panel E, median increase expression 9.8-fold 406 vs 5.4-fold, $\mathrm{p}=0.003$; Panel F, 69,306 pg/mL vs 15,232 pg/mL, $\mathrm{p}<0.0001$ ). Analyses by 407 Wilcoxon matched-pairs signed rank test. Bars indicate median values. Boxplots indicate 408 interquartile range and whiskers indicate minimum and maximum values. 
409 Figure 3. Concentrations of secreted IFN $\beta 1$, IFN- $\lambda 3$, and CXCL10 (normalized to total protein

410 concentration) in the supernatant of primary bronchial epithelial cell cultures 48 hours and 96

411 hours after infection with SARS-COV-2 (red circles) or HRV-16 (blue triangles). Secreted IFN $\beta 1$

412 concentrations peaked at 48 hours post viral infection (Panel A), and IFN $\beta 1$ concentrations

413 were significantly higher in HRV-16 infected cultures than SARS-CoV-2 infected cultures at both

414 time points (Panel A). IFN- 3 (Panel B) and CXCL10 (Panel C) concentrations were also

415 significantly greater in HRV-16 infected cultures at 48 and 96 hours following infection.

$416{ }^{*} \mathrm{p}<0.001,{ }^{\star *} \mathrm{p}=0.005,{ }^{\star \star *} \mathrm{p}=.0 .03,{ }^{\star \star \star *} \mathrm{p}=0.04, \# \mathrm{p}=0.2$. Analyses by Mann-Whitney tests.

417 Boxplots indicate interquartile range and whiskers indicate minimum and maximum values. 
418 Figure 4. Correlation between relative gene expression of IFNB1 or IFNL2 (normalized to

419 GAPDH) and SARS-CoV-2 viral replication by quantitative PCR in primary bronchial epithelial

420 cell cultures in children $(\mathrm{n}=14)$ and adults $(\mathrm{n}=8)$. IFNB1 and IFNL2 gene expression were

421 inversely correlated with SARS-CoV-2 replication (Panel A, Spearman $r=-0.61, p=0.003$; Panel

$422 B$, Spearman $r=-0.42, p=0.05)$. 
423 Figure 5. Correlation between secreted IFN $\beta 1$ concentration 48 hours after SARS-CoV-2

424 infection or CXCL10 concentration from the cell lysate 96 hours after SARS-CoV-2 infection

425 (normalized to total protein concentration) and 96-hour replication of SARS-CoV-2 by

426 quantitative PCR in primary bronchial epithelial cell cultures in children $(n=14)$ and adults $(n=8)$.

427 Secreted IFN 31 and SARS-CoV-2 replication were significantly inversely correlated (Panel A;

428 Spearman $r=-0.53, p=0.02)$, and $C X C L 10$ concentration from cell lysates was also significantly

429 inversely correlated with SARS-CoV-2 replication (Panel B; Spearman $r=-0.65, p=0.002$ ). 
430 Figure 6. SARS-CoV-2 replication by quantitative PCR in primary bronchial airway epithelial

431 cell cultures from children $(n=14)$ and adults $(n=10)$ infected in parallel with SARS-CoV-2 alone

432 at MOI=0.5 (red circles), SARS-CoV-2 infection 72 hours following pre-infection with HRV-16

433 ( $\mathrm{MOI}=0.5$ : blue triangles), pre- and concurrent treatment with recombinant IFNß1 (orange

434 squares), and pre- and concurrent treatment with recombinant IFN $\lambda 2$ (green diamonds). Viral

435 copy number was quantified by PCR in RNA harvested 96 hours after SARS-CoV-2 infection.

436 Pre-infection of primary bronchial AECs with HRV-16 significantly reduced SARS-CoV-2

437 replication (median copy number 267,264 vs $14,788, p=0.002$ ). Treatment of bronchial AEC

438 cultures with recombinant IFN $\beta 1$ or IFN $\lambda 2$ also significantly reduced SARS-CoV-2 replication

439 (median copy number 267,264 to $11,947, p=0.0001$; median copy number 267,264 to 11,856 ,

$440 \mathrm{p}=0.0002$, respectively). Kruskal-Wallis one-way ANOVA on ranks was used to compare all

441 experimental conditions. Dunn's test was used for comparisons between SARS-CoV-2 alone

442 and individual experimental conditions. Bars indicate median values. 
443 Figure 7. Relative gene expression of pattern-recognition receptor and RNA viral sensor IFIH1

444 (MDA5) (normalized to GAPDH expression) by primary bronchial airway epithelial cell cultures

445 in children $(n=14)$ and adults $(n=8)$. Gene expression was quantified by PCR in RNA harvested

44696 hours after parallel infection with SARS-CoV-2 (MOI=0.5, red diamonds) or HRV-16

447 (MOI=0.5, blue diamonds). IFIH1 expression was significantly higher after HRV-16 infection

448 than after SARS-CoV-2 infection ( $p=0.003$ by Wilcoxon matched-pairs signed rank test; bars

449 indicate median values). 
1. Medicine JHUa. Johns Hopkins University Coronavirus Resource Center - Mortality Analyses [Website]. [Available from: https://coronavirus.jhu.edu/data/mortality.

2. Lai CC, Liu YH, Wang CY, Wang YH, Hsueh SC, Yen MY, et al. Asymptomatic carrier state, acute respiratory disease, and pneumonia due to severe acute respiratory syndrome coronavirus 2 (SARS-CoV2): Facts and myths. Journal of microbiology, immunology, and infection = Wei mian yu gan ran za zhi. 2020.

3. Team CC-R. Severe Outcomes Among Patients with Coronavirus Disease 2019 (COVID-19) United States, February 12-March 16, 2020. MMWR Morbidity and mortality weekly report. 2020;69(12):343-6.

4. Cai J, Xu J, Lin D, Yang Z, Xu L, Qu Z, et al. A Case Series of children with 2019 novel coronavirus infection: clinical and epidemiological features. Clinical infectious diseases : an official publication of the Infectious Diseases Society of America. 2020.

5. Siegel DA, Reses HE, Cool AJ, Shapiro CN, Hsu J, Boehmer TK, et al. Trends in COVID-19 Cases, Emergency Department Visits, and Hospital Admissions Among Children and Adolescents Aged 0-17 Years - United States, August 2020-August 2021. MMWR Morbidity and mortality weekly report. 2021;70(36):1249-54.

6. Sampaio NG, Chauveau L, Hertzog J, Bridgeman A, Fowler G, Moonen JP, et al. The RNA sensor MDA5 detects SARS-CoV-2 infection. Scientific reports. 2021;11(1):13638.

7. Sa Ribero M, Jouvenet N, Dreux M, Nisole S. Interplay between SARS-CoV-2 and the type I interferon response. PLoS pathogens. 2020;16(7):e1008737.

8. Park A, Iwasaki A. Type I and Type III Interferons - Induction, Signaling, Evasion, and Application to Combat COVID-19. Cell Host Microbe. 2020;27(6):870-8.

9. Mazaleuskaya L, Veltrop R, Ikpeze N, Martin-Garcia J, Navas-Martin S. Protective role of Toll-like Receptor 3-induced type I interferon in murine coronavirus infection of macrophages. Viruses. 2012;4(5):901-23.

10. Fung SY, Yuen KS, Ye ZW, Chan CP, Jin DY. A tug-of-war between severe acute respiratory syndrome coronavirus 2 and host antiviral defence: lessons from other pathogenic viruses. Emerging microbes \& infections. 2020;9(1):558-70.

11. Mesel-Lemoine M, Millet J, Vidalain PO, Law H, Vabret A, Lorin V, et al. A human coronavirus responsible for the common cold massively kills dendritic cells but not monocytes. Journal of virology. 2012;86(14):7577-87.

12. Lau SKP, Lau CCY, Chan KH, Li CPY, Chen H, Jin DY, et al. Delayed induction of proinflammatory cytokines and suppression of innate antiviral response by the novel Middle East respiratory syndrome 
coronavirus: implications for pathogenesis and treatment. The Journal of general virology. 2013;94(Pt 12):2679-90.

13. Yoshikawa T, Hill TE, Yoshikawa N, Popov VL, Galindo CL, Garner HR, et al. Dynamic innate immune responses of human bronchial epithelial cells to severe acute respiratory syndrome-associated coronavirus infection. PloS one. 2010;5(1):e8729.

14. Niemeyer D, Mosbauer K, Klein EM, Sieberg A, Mettelman RC, Mielech AM, et al. The papain-like protease determines a virulence trait that varies among members of the SARS-coronavirus species. PLoS pathogens. 2018;14(9):e1007296.

15. Menachery VD, Gralinski LE, Mitchell HD, Dinnon KH, 3rd, Leist SR, Yount BL, Jr., et al. Middle East Respiratory Syndrome Coronavirus Nonstructural Protein 16 Is Necessary for Interferon Resistance and Viral Pathogenesis. mSphere. 2017;2(6).

16. Kindler E, Jonsdottir HR, Muth D, Hamming OJ, Hartmann R, Rodriguez R, et al. Efficient replication of the novel human betacoronavirus EMC on primary human epithelium highlights its zoonotic potential. mBio. 2013;4(1):e00611-12.

17. Sims AC, Tilton SC, Menachery VD, Gralinski LE, Schafer A, Matzke MM, et al. Release of severe acute respiratory syndrome coronavirus nuclear import block enhances host transcription in human lung cells. Journal of virology. 2013;87(7):3885-902.

18. Lei X, Dong X, Ma R, Wang W, Xiao X, Tian Z, et al. Activation and evasion of type I interferon responses by SARS-CoV-2. Nature communications. 2020;11(1):3810.

19. Blanco-Melo D, Nilsson-Payant BE, Liu WC, Uhl S, Hoagland D, Moller R, et al. Imbalanced Host Response to SARS-CoV-2 Drives Development of COVID-19. Cell. 2020;181(5):1036-45 e9.

20. Murphy RC, Lai Y, Barrow KA, Hamerman JA, Lacy-Hulbert A, Piliponsky AM, et al. Effects of Asthma and Human Rhinovirus A16 on the Expression of SARS-CoV-2 Entry Factors in Human Airway Epithelium. American journal of respiratory cell and molecular biology. 2020;63(6):859-63.

21. Ziegler CGK, Allon SJ, Nyquist SK, Mbano IM, Miao VN, Tzouanas CN, et al. SARS-CoV-2 Receptor ACE2 Is an Interferon-Stimulated Gene in Human Airway Epithelial Cells and Is Detected in Specific Cell Subsets across Tissues. Cell. 2020;181(5):1016-35 e19.

22. Zhang Q, Bastard P, Liu Z, Le Pen J, Moncada-Velez M, Chen J, et al. Inborn errors of type I IFN immunity in patients with life-threatening COVID-19. Science. 2020;370(6515).

23. Meffre E, Iwasaki A. Interferon deficiency can lead to severe COVID. Nature. 2020;587(7834):374-6.

24. Hadjadj J, Yatim N, Barnabei L, Corneau A, Boussier J, Smith N, et al. Impaired type I interferon activity and inflammatory responses in severe COVID-19 patients. Science. 2020;369(6504):718-24.

25. Ziegler CGK, Miao VN, Owings AH, Navia AW, Tang Y, Bromley JD, et al. Impaired local intrinsic immunity to SARS-CoV-2 infection in severe COVID-19. Cell. 2021;184(18):4713-33 e22. 
26. Vanderwall ERB, K.A.; Rich, L.M.; White, M.P.; Ziegler, S.F.; Rathe, J.A.; Debley, J.S. Interferon Responses by Differentiated Primary Bronchial Airway Epithelial Cells to SarsCoV2 Are Less Robust Than to Human Rhinovirus16. American Journal of Respiratory and Critical Care Medicine. 2021;203:A1292.

27. Lopez-Guisa JM, Powers C, File D, Cochrane E, Jimenez N, Debley JS. Airway epithelial cells from asthmatic children differentially express proremodeling factors. J Allergy Clin Immunol. 2012;129(4):9907.

28. Reeves SR, Kolstad T, Lien TY, Elliott M, Ziegler SF, Wight TN, et al. Asthmatic airway epithelial cells differentially regulate fibroblast expression of extracellular matrix components. The Journal of allergy and clinical immunology. 2014;134(3):663-70 e1.

29. Altman MC, Reeves SR, Parker AR, Whalen E, Misura KM, Barrow KA, et al. Interferon response to respiratory syncytial virus by bronchial epithelium from children with asthma is inversely correlated with pulmonary function. The Journal of allergy and clinical immunology. 2018;142(2):451-9.

30. James RG, Reeves SR, Barrow KA, White MP, Glukhova VA, Haghighi C, et al. Deficient Follistatinlike 3 Secretion by Asthmatic Airway Epithelium Impairs Fibroblast Regulation and Fibroblast-toMyofibroblast Transition. American journal of respiratory cell and molecular biology. 2018;59(1):104-13.

31. Barrow KA, Rich LM, Vanderwall ER, Reeves SR, Rathe JA, White MP, et al. Inactivation of Material from SARS-CoV-2-Infected Primary Airway Epithelial Cell Cultures. Methods Protoc. 2021;4(1).

32. Pfaffl MW. A new mathematical model for relative quantification in real-time RT-PCR. Nucleic acids research. 2001;29(9):e45.

33. Acharya D, Liu G, Gack MU. Dysregulation of type I interferon responses in COVID-19. Nat Rev Immunol. 2020;20(7):397-8.

34. Channappanavar R, Fehr AR, Vijay R, Mack M, Zhao J, Meyerholz DK, et al. Dysregulated Type I Interferon and Inflammatory Monocyte-Macrophage Responses Cause Lethal Pneumonia in SARS-CoVInfected Mice. Cell Host Microbe. 2016;19(2):181-93.

35. Liu G, Lee JH, Parker ZM, Acharya D, Chiang JJ, van Gent M, et al. ISG15-dependent activation of the sensor MDA5 is antagonized by the SARS-CoV-2 papain-like protease to evade host innate immunity. Nat Microbiol. 2021;6(4):467-78.

36. Snijder EJ, Limpens R, de Wilde AH, de Jong AWM, Zevenhoven-Dobbe JC, Maier HJ, et al. A unifying structural and functional model of the coronavirus replication organelle: Tracking down RNA synthesis. PLoS Biol. 2020;18(6):e3000715.

37. Romano M, Ruggiero A, Squeglia F, Maga G, Berisio R. A Structural View of SARS-CoV-2 RNA Replication Machinery: RNA Synthesis, Proofreading and Final Capping. Cells. 2020;9(5).

38. Min YQ, Huang $M$, Sun X, Deng F, Wang H, Ning YJ. Immune evasion of SARS-CoV-2 from interferon antiviral system. Comput Struct Biotechnol J. 2021;19:4217-25. 
39. Scutigliani EM, Kikkert M. Interaction of the innate immune system with positive-strand RNA virus replication organelles. Cytokine Growth Factor Rev. 2017;37:17-27.

40. Hackbart M, Deng X, Baker SC. Coronavirus endoribonuclease targets viral polyuridine sequences to evade activating host sensors. Proc Natl Acad Sci U S A. 2020;117(14):8094-103.

41. Kindler E, Gil-Cruz C, Spanier J, Li Y, Wilhelm J, Rabouw HH, et al. Early endonuclease-mediated evasion of RNA sensing ensures efficient coronavirus replication. PLoS pathogens. 2017;13(2):e1006195.

42. Deng X, Hackbart M, Mettelman RC, O'Brien A, Mielech AM, Yi G, et al. Coronavirus nonstructural protein 15 mediates evasion of dsRNA sensors and limits apoptosis in macrophages. Proc Natl Acad Sci U S A. 2017;114(21):E4251-E60.

43. Oh SJ, Shin OS. SARS-CoV-2 Nucleocapsid Protein Targets RIG-I-Like Receptor Pathways to Inhibit the Induction of Interferon Response. Cells. 2021;10(3).

44. Guo G, Gao M, Gao X, Zhu B, Huang J, Luo K, et al. SARS-CoV-2 non-structural protein 13 (nsp13) hijacks host deubiquitinase USP13 and counteracts host antiviral immune response. Signal Transduct Target Ther. 2021;6(1):119.

45. Zheng $\mathrm{Y}$, Zhuang MW, Han L, Zhang J, Nan ML, Zhan $\mathrm{P}$, et al. Severe acute respiratory syndrome coronavirus 2 (SARS-CoV-2) membrane (M) protein inhibits type I and III interferon production by targeting RIG-I/MDA-5 signaling. Signal Transduct Target Ther. 2020;5(1):299.

46. Wu J, Shi Y, Pan X, Wu S, Hou R, Zhang Y, et al. SARS-CoV-2 ORF9b inhibits RIG-I-MAVS antiviral signaling by interrupting K63-linked ubiquitination of NEMO. Cell Rep. 2021;34(7):108761.

47. Yuen CK, Lam JY, Wong WM, Mak LF, Wang X, Chu H, et al. SARS-CoV-2 nsp13, nsp14, nsp15 and orf6 function as potent interferon antagonists. Emerging microbes \& infections. 2020;9(1):1418-28.

48. Fu YZ, Wang SY, Zheng ZQ, Yi H, Li WW, Xu ZS, et al. SARS-CoV-2 membrane glycoprotein M antagonizes the MAVS-mediated innate antiviral response. Cell Mol Immunol. 2021;18(3):613-20.

49. Wu Y, Ma L, Zhuang Z, Cai S, Zhao Z, Zhou L, et al. Main protease of SARS-CoV-2 serves as a bifunctional molecule in restricting type I interferon antiviral signaling. Signal Transduct Target Ther. 2020;5(1):221.

50. Shin D, Mukherjee R, Grewe D, Bojkova D, Baek K, Bhattacharya A, et al. Papain-like protease regulates SARS-CoV-2 viral spread and innate immunity. Nature. 2020;587(7835):657-62.

51. Klemm T, Ebert G, Calleja DJ, Allison CC, Richardson LW, Bernardini JP, et al. Mechanism and inhibition of the papain-like protease, PLpro, of SARS-CoV-2. EMBO J. 2020;39(18):e106275.

52. Jiang HW, Zhang HN, Meng QF, Xie J, Li Y, Chen H, et al. SARS-CoV-2 Orf9b suppresses type I interferon responses by targeting TOM70. Cell Mol Immunol. 2020;17(9):998-1000.

53. Gordon DE, Jang GM, Bouhaddou M, Xu J, Obernier K, White KM, et al. A SARS-CoV-2 protein interaction map reveals targets for drug repurposing. Nature. 2020;583(7816):459-68. 
54. Miorin L, Kehrer T, Sanchez-Aparicio MT, Zhang K, Cohen P, Patel RS, et al. SARS-CoV-2 Orf6 hijacks Nup98 to block STAT nuclear import and antagonize interferon signaling. Proc Natl Acad Sci U S A. 2020;117(45):28344-54.

55. Xia H, Cao Z, Xie X, Zhang X, Chen JY, Wang H, et al. Evasion of Type I Interferon by SARS-CoV-2. Cell Rep. 2020;33(1):108234.

56. Mu J, Fang $\mathrm{Y}$, Yang $\mathrm{Q}$, Shu $\mathrm{T}$, Wang $\mathrm{A}$, Huang $\mathrm{M}$, et al. SARS-CoV-2 N protein antagonizes type I interferon signaling by suppressing phosphorylation and nuclear translocation of STAT1 and STAT2. Cell Discov. 2020;6:65.

57. Naslavsky MS, Vidigal M, Matos L, Coria VR, Batista Junior PB, Razuk A, et al. Extreme phenotypes approach to investigate host genetics and COVID-19 outcomes. Genet Mol Biol. 2021;44(1 Suppl 1):e20200302.

58. Cheemarla NR, Watkins TA, Mihaylova VT, Wang B, Zhao D, Wang G, et al. Dynamic innate immune response determines susceptibility to SARS-CoV-2 infection and early replication kinetics. J Exp Med. 2021;218(8).

59. Dee K, Goldfarb DM, Haney J, Amat JAR, Herder V, Stewart M, et al. Human Rhinovirus Infection Blocks Severe Acute Respiratory Syndrome Coronavirus 2 Replication Within the Respiratory Epithelium: Implications for COVID-19 Epidemiology. The Journal of infectious diseases. 2021;224(1):31-8.

60. Monk PD, Marsden RJ, Tear VJ, Brookes J, Batten TN, Mankowski M, et al. Safety and efficacy of inhaled nebulised interferon beta-1a (SNG001) for treatment of SARS-CoV-2 infection: a randomised, double-blind, placebo-controlled, phase 2 trial. The Lancet Respiratory medicine. 2021;9(2):196-206. 


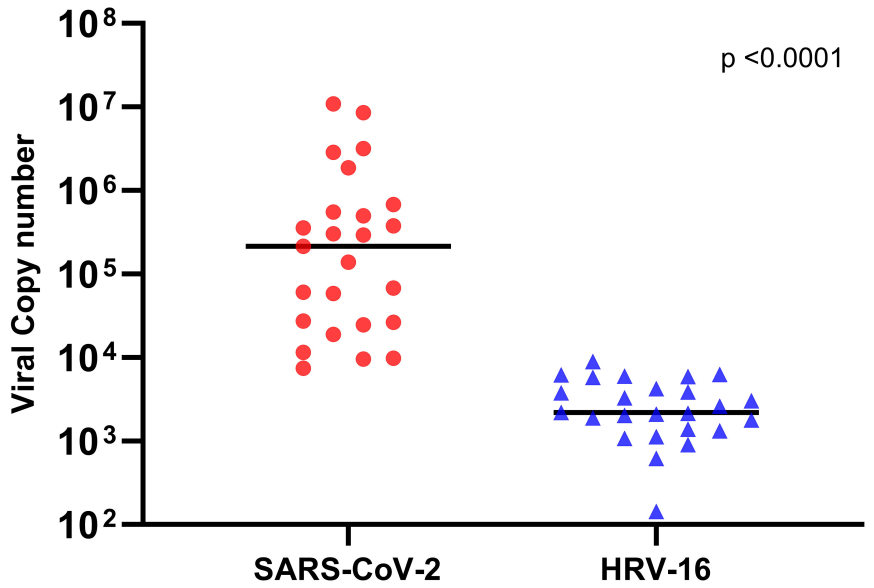


AFNB1 expression in response to
SARS-CoV-2 vs. HRV-16

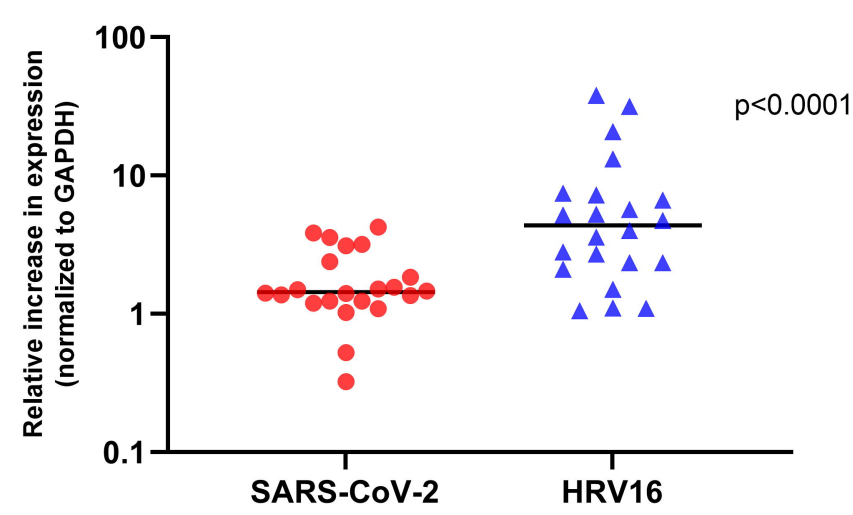

C. IFNL2 expression in response to

SARS-CoV-2 vs. HRV-16

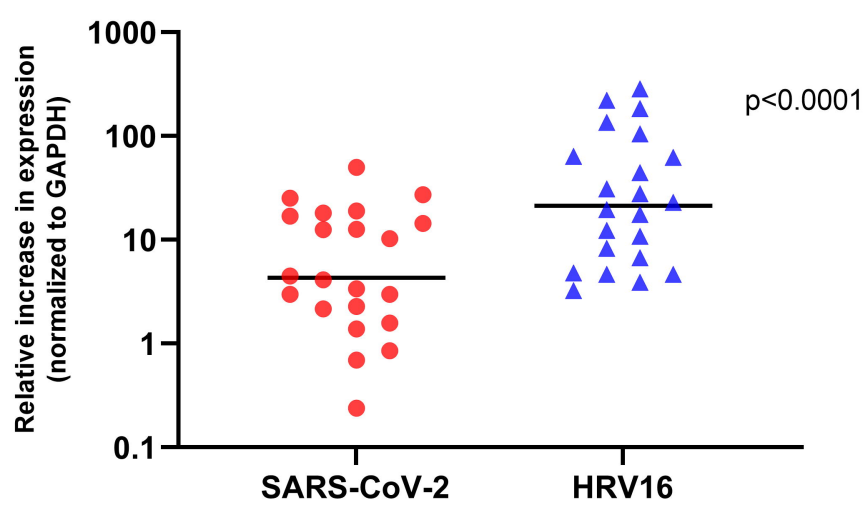

E CXCL10 expression in response to SARS-CoV-2 vs. HRV-16

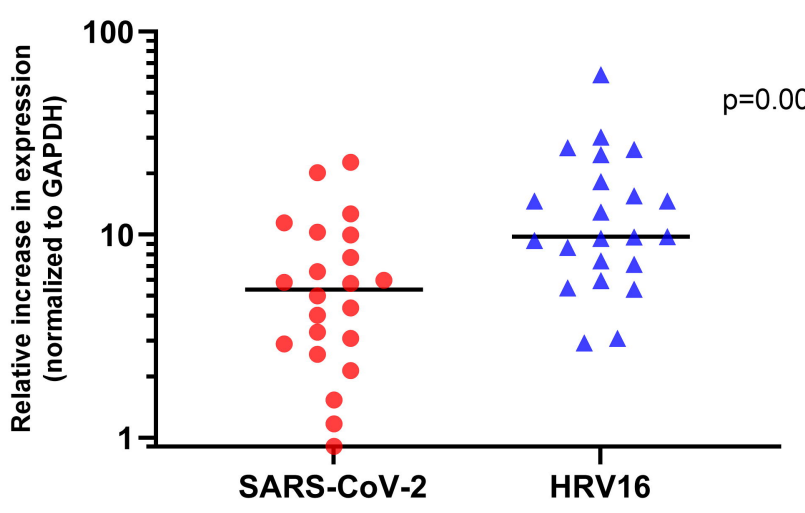

B. IFN $\beta 1$ protein cell lysate SARS-CoV-2 vs. HRV-16

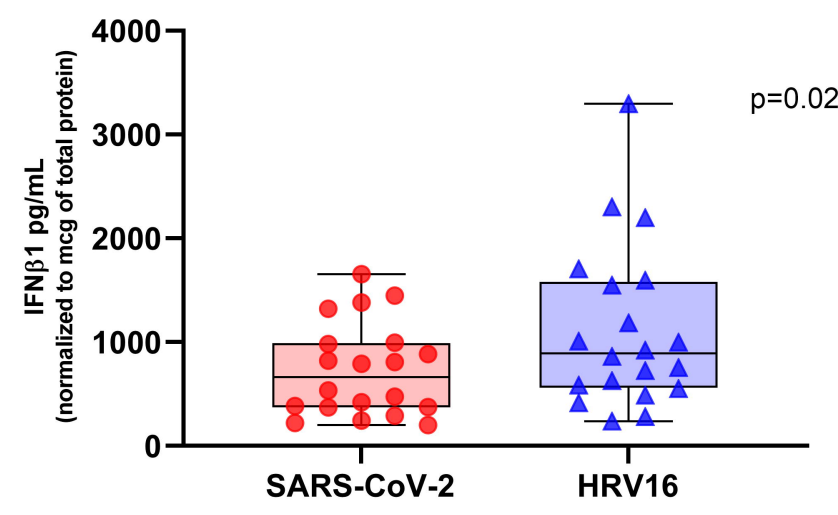

D. IL-28a/IFN- $\lambda 2$ protein concentration SARS-CoV-2 vs. HRV-16

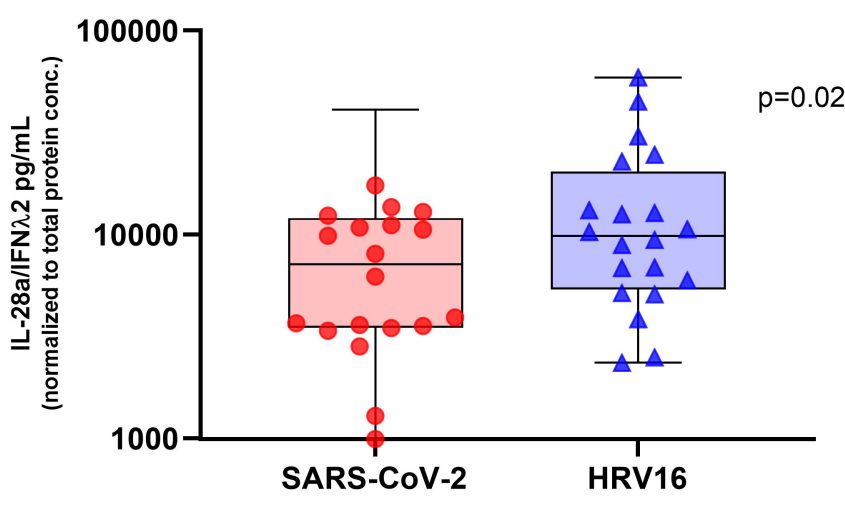

F. CXCL-10 protein concentration SARS-CoV-2 vs. HRV-16

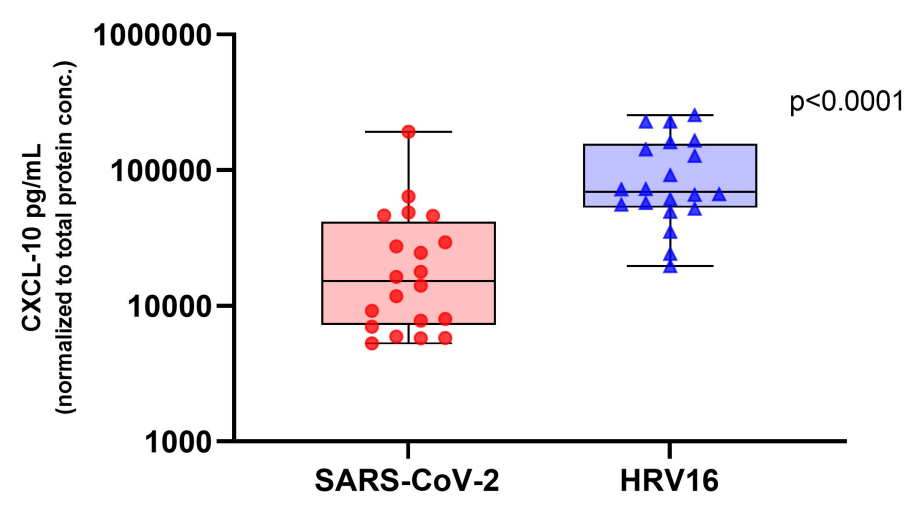


A.

Secreted IFN $\beta 1$

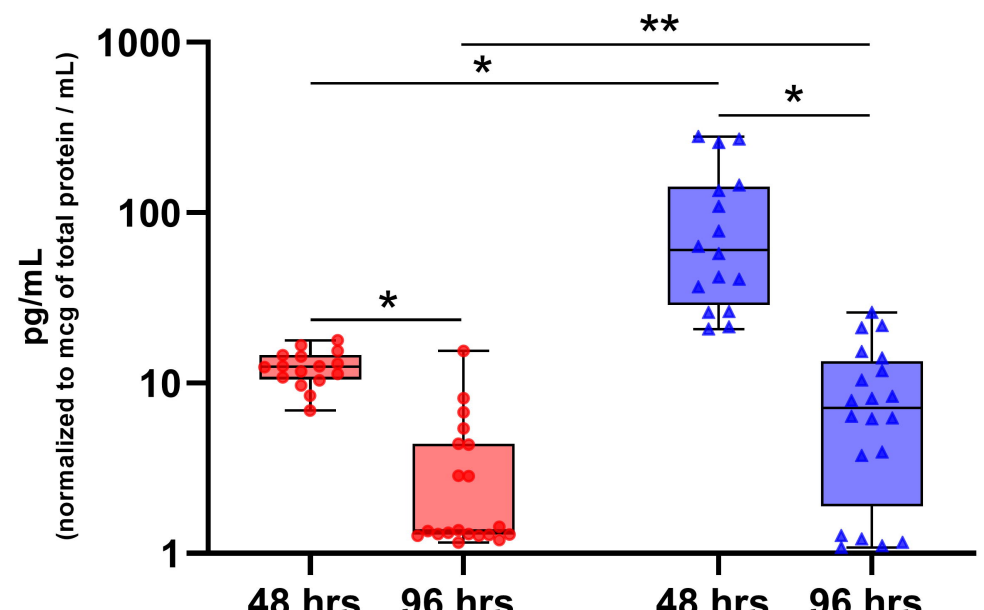

B.

Secreted IFN $\lambda 3$

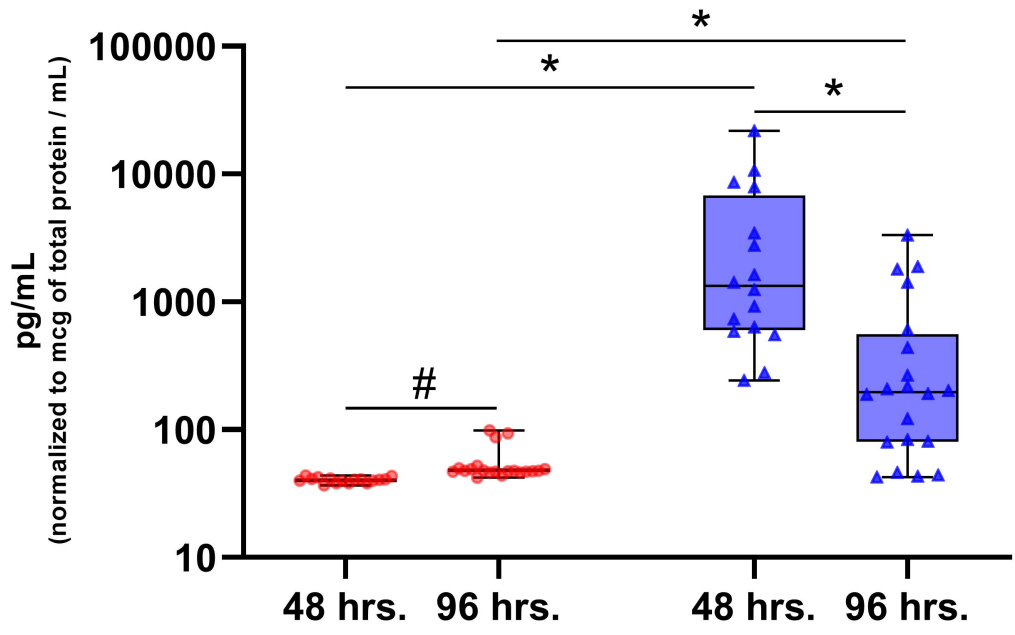

c.

Secreted CXCL10

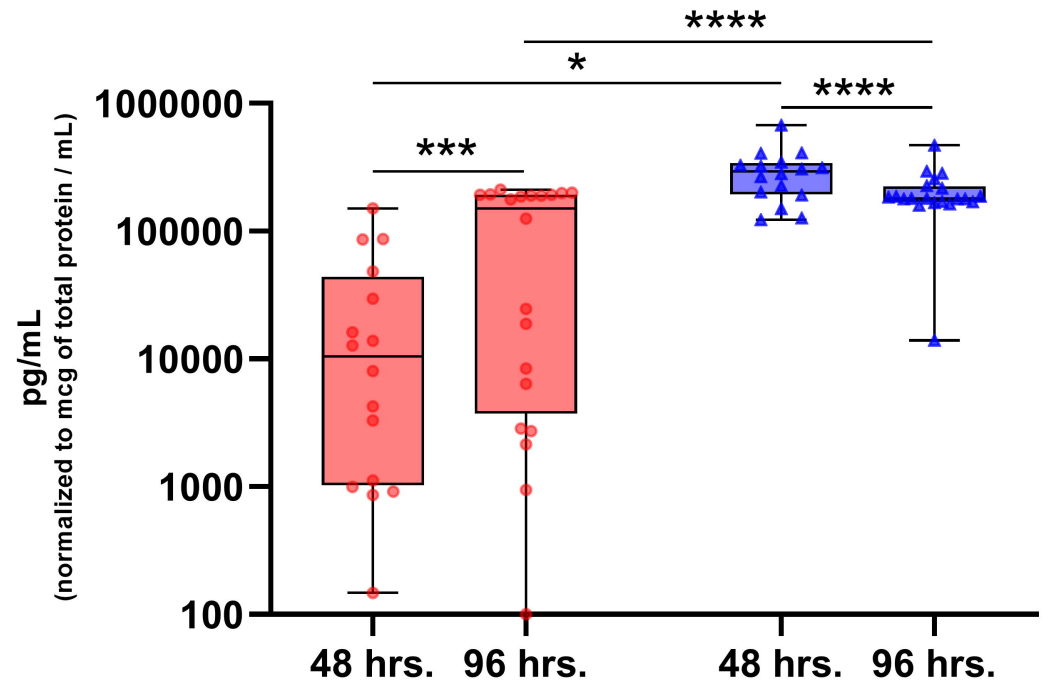


A. Correlation between IFNB1 expression and SARS-CoV-2 replication (96hrs.)

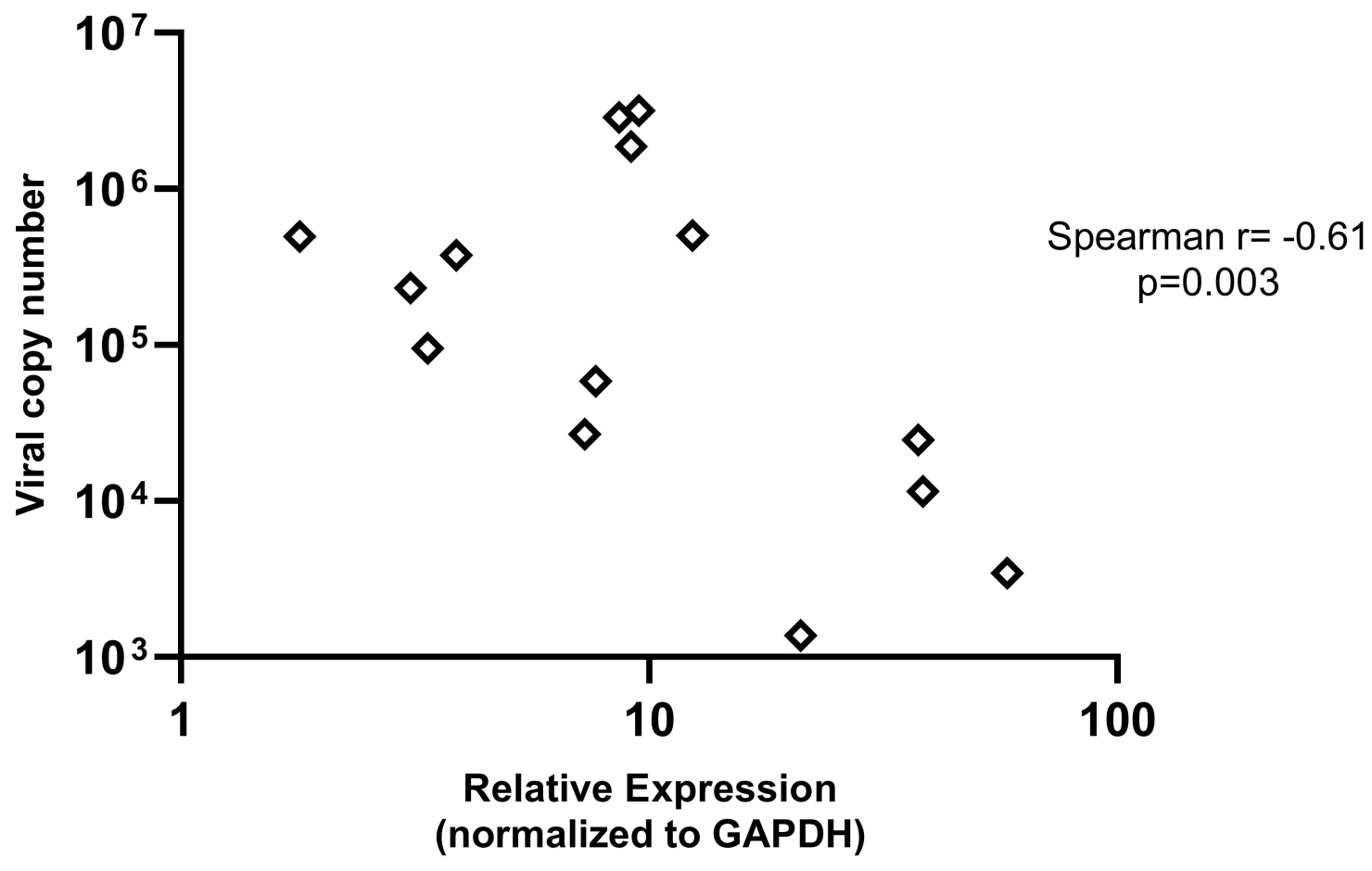

B. Correlation between IFNL2 expression
and SARS-CoV-2 replication (96hrs.)

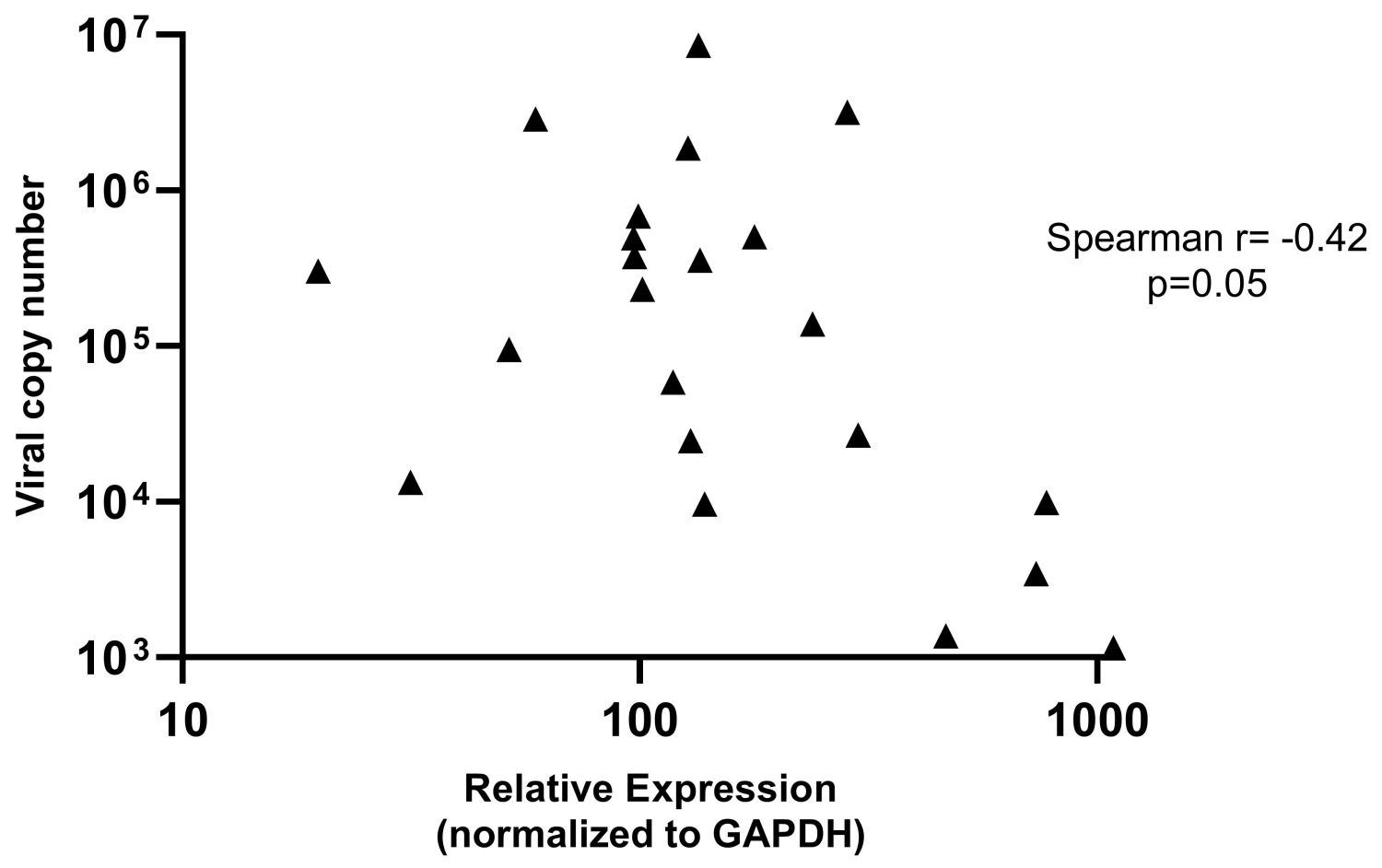


A. Correlation between IFN $\beta 1$ protein in supernatant (48hrs.) and SARS-CoV-2 replication

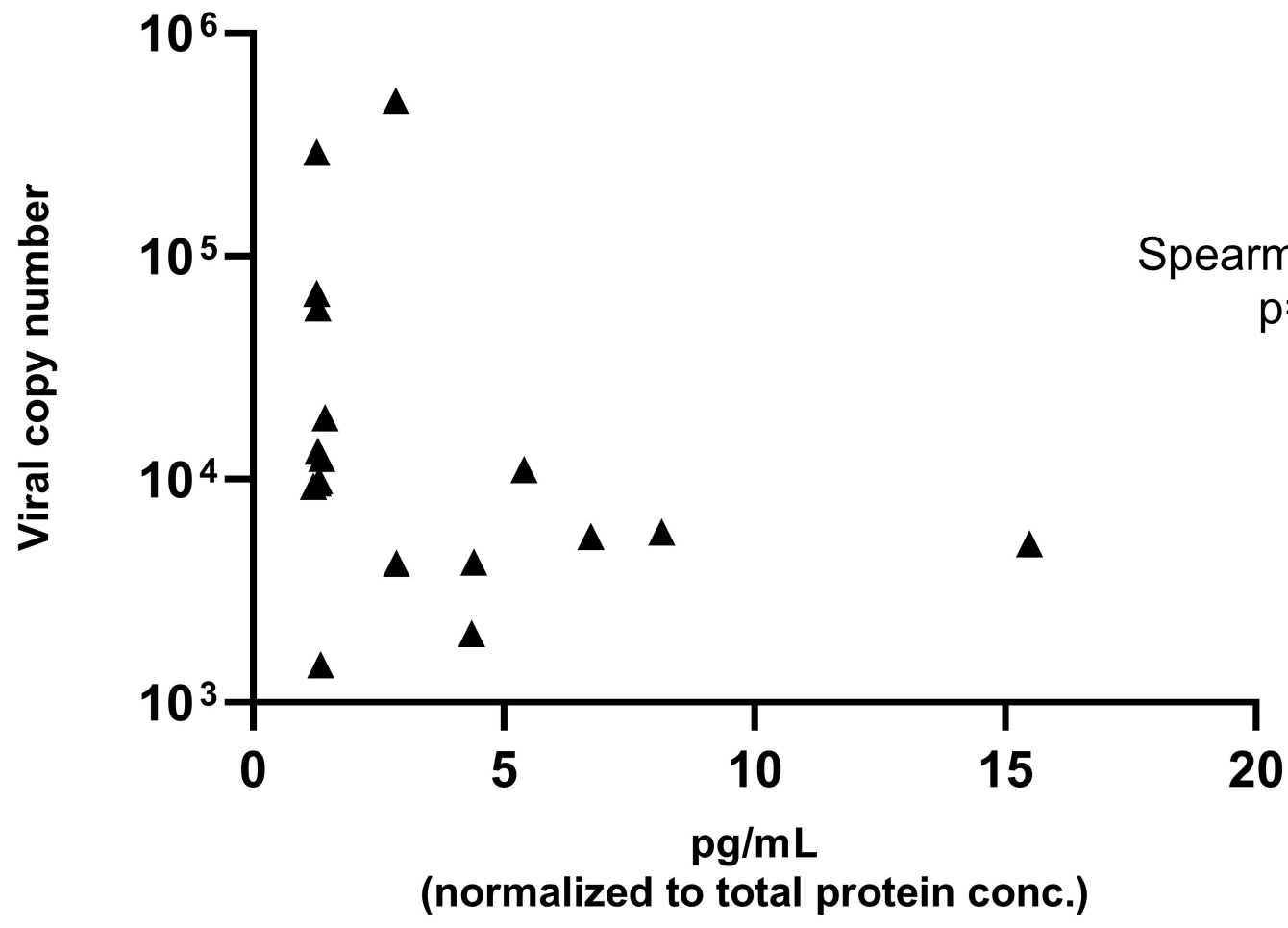

B. Correlation between CXCL10 protein in cell lysate and SARS-CoV-2 replication

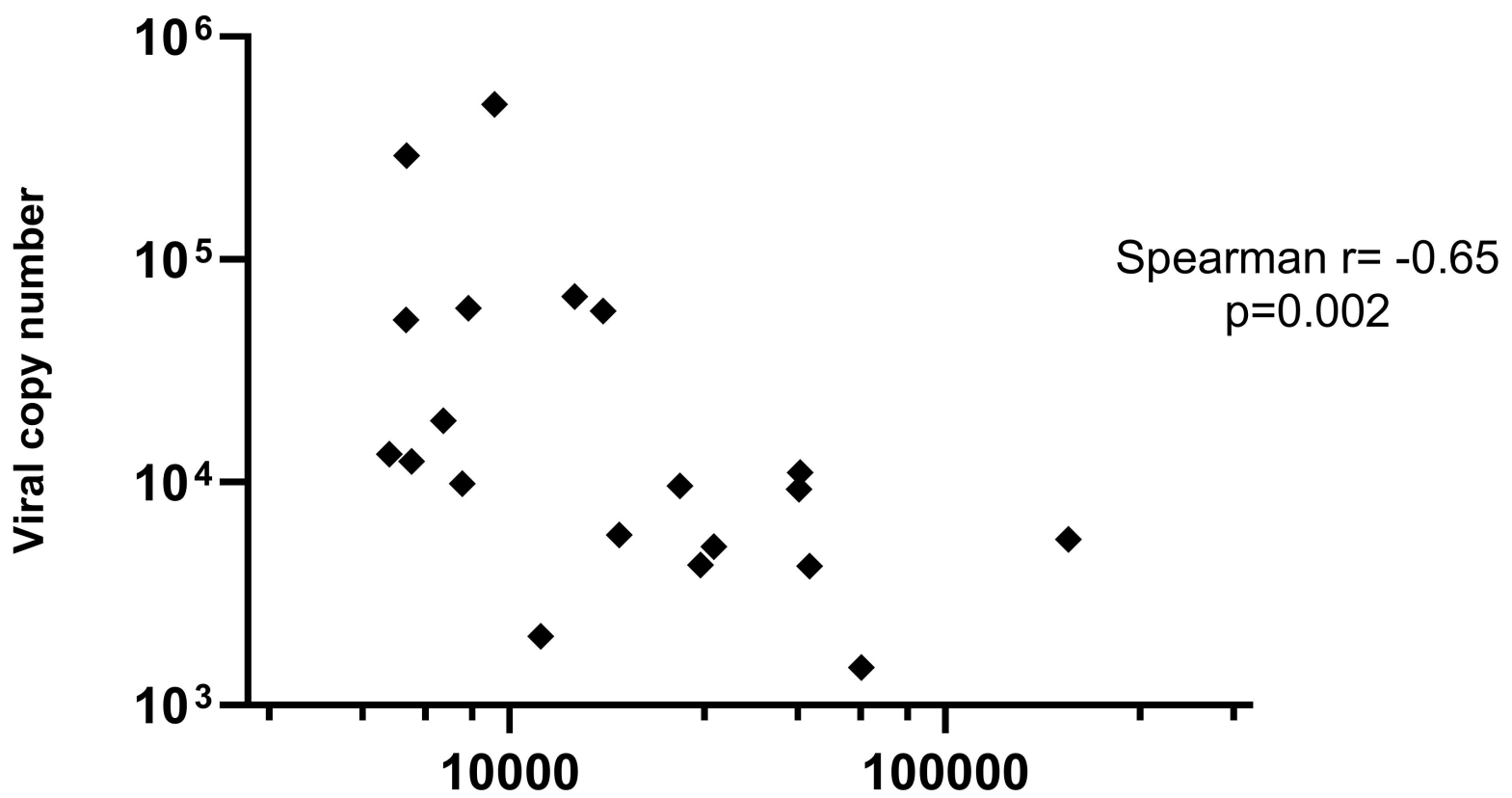

$\mathrm{pg} / \mathrm{mL}$

(normalized to total protein conc.) 


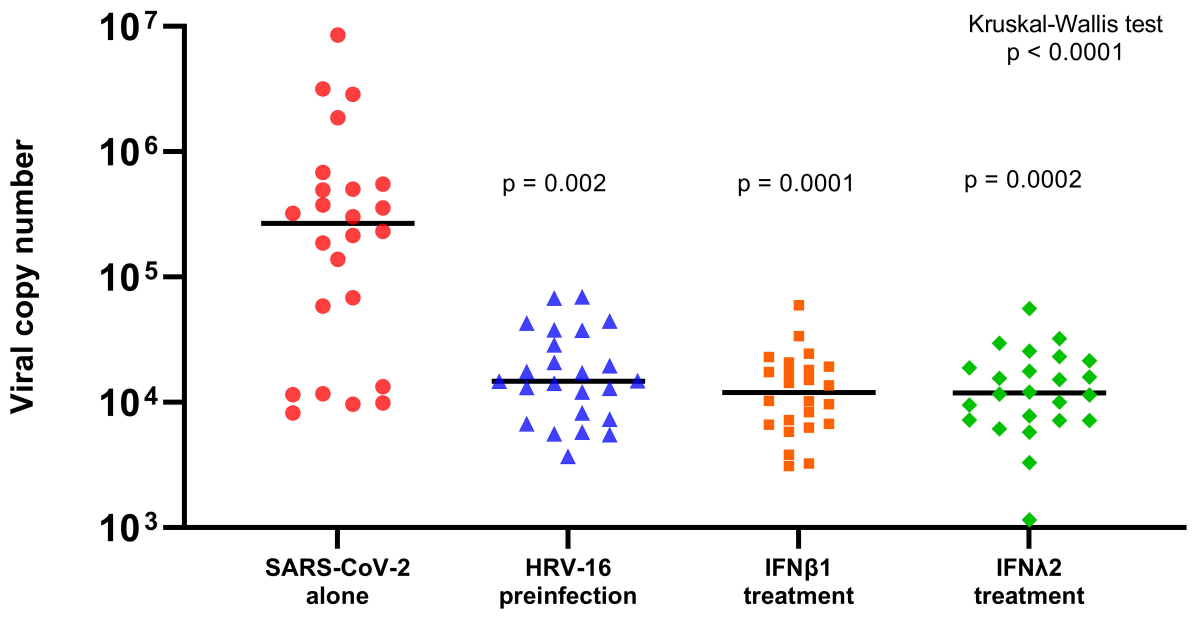




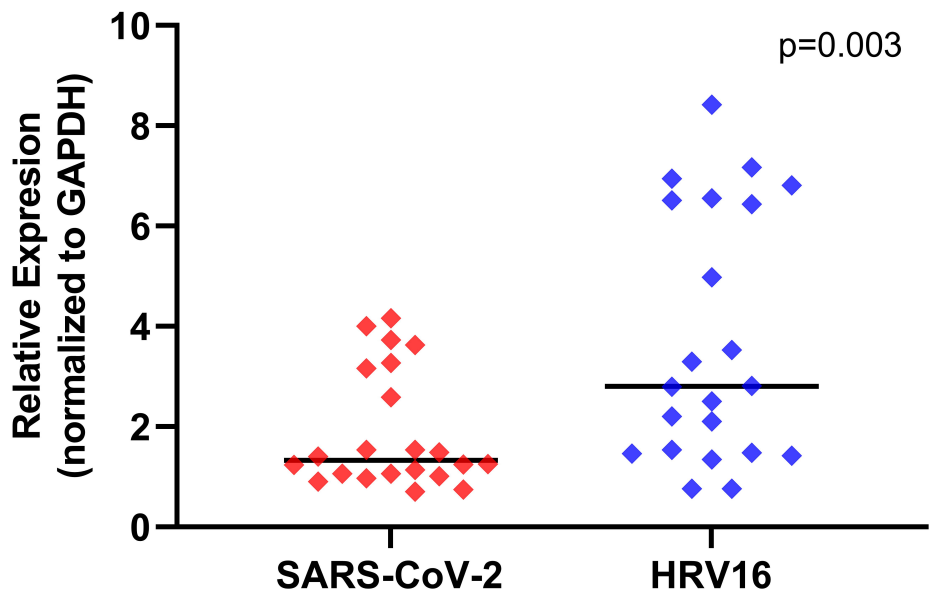

